\title{
Experiment on determination of ethanol chemical shift and concentration of ethanol solution based on nuclear magnetic resonance instrument
}

\author{
Liang Xinyi ${ }^{1, *}$ \\ ${ }^{1}$ Department of Food Science and Engineering, Beijing Forestry University, Beijing 100083, China
}

\begin{abstract}
In this designing experiment, a nuclear magnetic resonance instrument was used to study the chemical shift properties of ethanol based on the principle of magnetic shielding effect. At the same time, the nuclear magnetic resonance method was used to study the transverse relaxation time and signal strength of ethanol aqueous solution in the range of $5 \%$ to $40 \%$. The relationship between different mass fraction of ethanol-aqueous solution and nuclear magnetic resonance signal intensity, relaxation time and solution concentration were analysed. According to the results, due to the limitation of experimental instruments, no regular changes were observed. The results show that the addition of copper sulphate can increase the ethanol content and the effect of the ethanol content on the lateral relaxation time: the ethanol content varies between $5 \%$ and $40 \%$, and the lateral relaxation time changes from $8.42 \mathrm{~ms}$ to $40.35 \mathrm{~ms}$. According to the experimental results, the empirical formulas of the concentration and lateral relaxation time of ethanol aqueous solution with fixed amount of copper sulphate were fitted. Using this relationship, the NMR measurement of the concentration of ethanol-water solution can be achieved, which provides a new method for the convenient and accurate measurement of the concentration of ethanol solution.
\end{abstract}

\section{Introduction}

NMR (Nuclear Magnetic Resonance, NMR) spectroscopy is not only a very rapidly developing science, but also a modern technology that can penetrate into the material without destroying the structure of the measured object. It can obtain information about the nucleus by using the energy change of the nucleus in the magnetic field. It has the advantages of rapid, accurate and high resolution, so it has been widely used in scientific research and production.

Nuclear magnetic resonance (NMR) is a new technology which can cause energy level splitting under the action of magnetic field. If there is a corresponding RF magnetic field, it will cause resonance transition between nuclear energy levels. The experiment was first confirmed in 1946 by Harvard University's Bassel (E.M. Purcell) and Stanford University's Bloch (F. Bloch) et al. They shared the 1952 Nobel Prize in Physics. Nuclear magnetic resonance spectroscopy has been developed as a mature, conventional method for analyzing molecular structures and the characterization of physical and chemical properties of substances, and has found wide applications in fields of chemistry, biology, medicine, materials, etc, benefitting from its features of noninvasion, high resolution and high-throughput information. Recently, a high level of interest has reemerged in the development of pure shift NMR techniques which aim for significantly improving spectral resolution in overlapped regions. ${ }^{[1]}$ Since the 1970s, on the basis of magnetic resonance spectroscopy and computed tomography, a new medical diagnostic technique, nuclear magnetic resonance imaging, has been developed and has achieved great success clinically. Because the electron cloud outside the spin core adds a static magnetic field $\mathrm{B}_{0}$, the magnetic shielding effect of a substance produces a chemical shift. Using nuclear magnetic resonance imaging technology, chemical displacement imaging has an important application in medicine. For the chemical shift of ethanol, the dispersion of anhydrous ethanol in tumor by chemical shift imaging can be used to reflect the lesion signal. ${ }^{[2]}$

The NMR chemical shift is highly sensitive to molecular geometry, hydrogen bonding, solvent, temperature, and a few other factors. When the magnetic shielding constant is calculated, it can be converted to chemical shifts, which is considered valuable aids in NMR peak assignment. This gives elaborated information on molecular geometry and intermolecular effects. Calculating chemical shifts in solution is very complicated for it requires consideration of solvent effects and conformational averaging. ${ }^{[3]}$ Pennanen et al ${ }^{[4]}$ presented a general and systematic theory of electronic structure regarding nuclear magnetic resonance shielding tensors of paramagnetic atoms, molecules and non-

*evelyn_liang@bjfu.edu.cn 
metallic solids and their related chemical shifts. The theory is verified by first principles calculations of organometallic molecules. A method that is fast and easy for calculating NMR chemical shieldings of crystalline solids is proposed by Drainsky. ${ }^{[5]}$ The method combines the advantages of both traditional approaches, including periodic calculations using plane-wave basis sets as well as molecular computing approaches. The periodic calculations capture the periodic nature of crystalline solids, but the computational level of the electronic structure calculation is limited to general-gradientapproximation (GGA) density functionals. The results show that correcting the GGA result calculated for molecules separated at higher theoretical levels can significantly improve the correlation between experimental and calculated chemical shifts, while adding little additional computational cost. The corrections calculated with the mixed density function greatly improve the accuracy of chemical shift predictions for ${ }^{13} \mathrm{C},{ }^{15} \mathrm{~N}$, and ${ }^{17} \mathrm{O}$ and can identify errors in currently published experimental data. Marell et al ${ }^{[5]}$ has reported a study on NMR chemical shift, discussing conformationally challenging seven-membered lactones and comparing computed and experimental data sets. Calculations included a complete conformational analysis of each lactone, Boltzmann-weighted averaging of the chemical shifts of all conformers, and a linear correction of the calculated chemical shifts, with the results pointing out that the former gives a better basis to distinguish these diastereomers.

Bowen et al ${ }^{[6]}$ proposed a scheme that, with the exception of merely monitoring the reaction process, also used real-time, hyperpolarization enhanced nuclear magnetic resonance to obtain time correlation. Because even if spin-bearing atoms directly participate in a reaction, the spin state of the nucleus can remain unchanged, so it is possible to link the positions of these atoms between the reactant and product species over time. The application of this technology in Grignard addition reaction of methylmagnesium bromide and 3methylbenzophenone was studied. In the fields of organic chemistry, enzymology, or protein folding, the same experiment can also be used to determine the mechanism and intermediate state of non-equilibrium processes. Shifts are usually obtained by titrating different concentrations of ligands to a fixed concentration of receptors. Among other things, they can be used to determine affinity constants, locate binding surfaces, or distinguish binding mechanisms.

Sundell et al ${ }^{[7]}$ analyzed the sign of the ${ }^{15} \mathrm{~N} /{ }^{1} \mathrm{H}$ shift changes when Scribble's PDZ1 domain interacted with two pairs of phosphorylated and unphosphorylated peptides. The results show that the difference in the molecular basis of the PDZ-ligand interaction can be obtained from our analysis, and the classical chemical shift perturbation analysis method is not sensitive to it. Besides, the correlation between affinity and millisecond movement was also discovered. Applying this method to Cyclophilin, cis-trans isomerase revealed the molecular details of peptide recognition. Directional vector chemical shift analysis is a method to identify the molecular origin of a class of ligand-like binding specificities and is often used in drug discovery. Lundborg et al ${ }^{[8]}$ stated that CASPER, the acronym for computer-aided spectroscopic evaluation of polysaccharides, applies liquid nuclear magnetic resonance data to elucidating the carbohydrate structure based on the predicted ${ }^{1} \mathrm{H}$ and ${ }^{13} \mathrm{C}$ chemical shifts. Taking oligosaccharides and bacterial polysaccharides as examples, it was proved that the development based on the multiple through-bond J-based correlation significantly enhanced the credibility of the sequence connection proposed in the analysis. This method can also be used for predicting ${ }^{1} \mathrm{H}$ and ${ }^{13} \mathrm{C}$ NMR chemical shifts of synthetic oligosaccharides and glycoconjugates, thus confirming the proposed structure.

In addition, ethanol-water mixing system is also widely used in food, medicine, fermentation and other fields, water and ethanol are strong polar molecules, they can exist by hydrogen bonding with different cluster association structure. When the two are mixed in different proportions, it is not simple physical mixing, and the cluster association state will change accordingly, that is, the physical and chemical environment of the two changes. The research of this system has attracted the attention of many scholars at home and abroad. various theories and detection methods have been used to study the intermolecular interactions within the system and to measure the ethanol concentration. Among them, NMR is an important method to study ethanol-water mixing system. This experiment successfully reveals to the experimenters the principle of pulsed NMR and its relaxation time and chemical shift. Zarzycki et al ${ }^{[9]}$ used density functional methods to calculate the Gaugeinvariant NMR chemical shifts of the $\mathrm{C}$ and $\mathrm{H}$ sites in ethanol in various conformations and solvated environments. The effects of different exchange and related functions as well as different basis sets are studied systematically. Ghanghas et al ${ }^{[10]}$ outlined a simple procedure that uses proton nuclear magnetic resonance spectroscopy to establish the hydrogen bond geometry in liquid ethanol, and distinguishes the methyl group of $\mathrm{CH}_{3} \mathrm{CH}_{2} \mathrm{OH}$ by using the difference in the proton chemical shift value generated by the secondary isotope effect The line of deuterated $\mathrm{CH}_{3} \mathrm{CD}_{2} \mathrm{OH}$ in the ${ }^{1} \mathrm{H}$ NMR spectrum of the mixture of both and hydroxyl protons. An interesting finding emerging from the study is that the linear relationship between the distance and angle of hydrogen bonding in ethanol.

This experiment successfully revealed to the experimenter the principles of pulsed nuclear magnetic resonance and its relaxation time and chemical shift. The ingenious application of the amplification effect of copper ions $\left(\mathrm{Cu}^{2+}\right)$ on the local magnetic field to study the ethanol aqueous solution effectively reduced the sample $T_{2}$ and improved the The feasibility of detecting the content of ethanol aqueous solution in low field nuclear magnetic resonance technology. ${ }^{[1]}$

\section{Experimental Section}

The construction and geometrical dimensions of the composite slabs are shown in Fig.1. It is noted that the 
slab specimens consist of the thin-walled steel skeleton which also is formed with H-type main steel beams and steel channels, lightweight precast panels set upon the steel skeleton, shear keys connected to the main steel beams and post-pouring concrete layer.

It is especially noted that the geometrical dimensions of cross-section of the thin-walled steel beams, steel channels and dimensions of the precast panels are shown in Fig.2. It is seen that the thickness of steel plate of the main steel beams and the steel channels is $0.6 \mathrm{~mm}$, and thickness of the precast panels is $40 \mathrm{~mm}$, and depth of groove of the precast panel is $10 \mathrm{~mm}$.

\subsection{Experiment content}

a. Chemical shift of ethanol solution

b. Relationship between ethanol solution concentration and lateral relaxation time $\mathrm{T}_{2}$

c. Relationship between concentration of ethanol solution and nuclear magnetic resonance signal intensity

\subsection{Experimental program}

a. Measurement of chemical shift in ethanol

1. Use hard pulse FID to find the center frequency.

2. Peak picking, record the chemical shift of different peaks.

3. Use the peak position measurement function to measure the height of the spectral peaks in sequence and record the values.

4. Measure the half-height width of the spectral peaks in sequence and calculate the resolution.

b. Relationship between ethanol solution concentration and lateral relaxation time $\mathrm{T}_{2}$

1. Use the spin echo method to study different concentrations.

2. Record, perform polynomial fitting, get empirical equations, and calculate correlation coefficients.

3. Use the obtained formula, the horizontal relaxation time of a group of ethanol solutions of unknown concentration is measured, and the concentration is reversed to compare the true value.

c. Relationship between concentration of ethanol solution and signal intensity

1. Define the peak area as the total intensity of the signal peak, then conclude the relationship between the nuclear magnetic resonance signal intensity and the ethanol solution mass fraction.

2. Use the obtained formula to measure the lateral relaxation time of a group of ethanol solutions of unknown concentration, inversely their concentration, and compare their true values.

\subsection{Experimental samples}

In this experiment, an ethanol solution containing solute with a concentration (mass fraction) of $5 \% \mathrm{CuSO}_{4}$ was used for the experiment. The steps to configure the solution are as follows. (taking an ethanol solution with a mass fraction of $20 \%$ as an example)

1. The original quality of the weighing container $\mathrm{m}_{0}$

2. Add copper sulfate solids until the scale reads 0.4 grams $+\mathrm{m}_{0}$

3. Add water until the mass is 16 grams $+m_{0}$

4. Add absolute ethanol until the mass is 20 grams $+m_{0}$

5. Shake the container to dissolve the copper sulfate completely

6. Use a plastic tip dropper to take 3 grams sample into the test tube for measurement

\section{Test Results and Discussions}

\subsection{Measurement of ethanol chemical shift}

Static loading tests of three composite slab specimens as shown in Fig.1 are finished. The loading point and the measuring points of displacements are shown in Fig.3.

The specimens are denoted as SJ1, SJ2 and SJ3, for which the constraint conditions for them are different. In particularly, two ends of the main steel beams of specimens SJ1 and SJ2 are all simply supported, and four corners of specimen $\mathrm{SJ} 2$ are restrained in vertical direction, however two ends of the main steel beam of specimen SJ3 are fixed support. All the other parameters of three specimens are the same with each other as shown above. Loading scene of the specimens is shown in Fig.4. It is denoted that the measuring point of the relative displacement shown in Fig.3(a) is used to measure the horizontal displacement of concrete panel at hinged-fixed end of the main beam, that is the horizontal slip of the concrete panel to the steel beam. The measuring points 1-1 and 1-2 of strains as shown in Fig.3(c) are located on the upper flange of main steel beam, and the final strain of the upper flange of steel beam takes the mean value of the two strains at two points, and denoted as 1 . All the other measuring points of strains of the cross section of the steel beam are denoted in the same way.

Ethanol has three peaks under NMR. The root cause is the magnetic shielding effect of the electron cloud outside the spin nucleus and the net magnetic field B0. Considering the magnetic shielding effect of the electron cloud, the actual magnetic field strength of the nucleus (proton) is

$$
\mathrm{B}_{\text {nucleus }}=\mathrm{B}_{0}-\sigma \mathrm{B}_{0}=\mathrm{B}_{0}(1-\sigma)
$$

In the formula, $\sigma$ is the shielding coefficient.

Therefore, the nucleus in the molecule has different chemical bonds with other atoms, and the shielding coefficient $\sigma$ is also different, which causes their resonance frequency to be different.

$$
\omega_{0}=\gamma(1-\sigma) B_{0}
$$


The chemical shift can be measured by the frequency offset, but the resonance frequency changes with the external field 0 B. Such a measurement is obviously inconvenient. The actual chemical shift is represented by a dimensionless $\delta$ in ppm.

$\delta=\frac{\sigma_{B}-\sigma_{S}}{1-\sigma_{S}} \times 10^{6} \approx\left(\sigma_{R}-\sigma_{S}\right) \times 10^{6}$
In the formula, $\sigma_{R}$ and $\sigma_{S}$ are the shielding coefficients of the reference object and the sample.

The molecular formula of ethanol contains one $\mathrm{OH}$ bond, one $\mathrm{CH}_{2}$ bond, and $\mathrm{CH}_{3}$ bond. The chemical shift is shown in Fig.1.

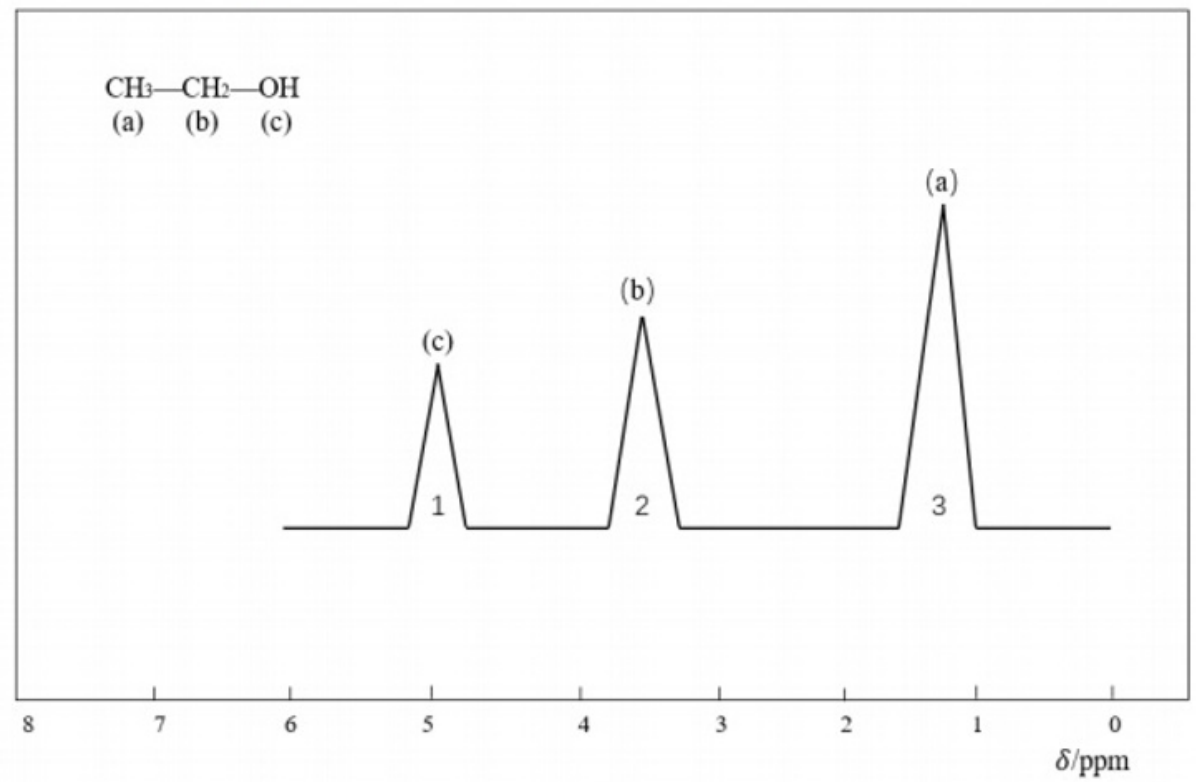

Fig .1 Ethanol molecular structure and magnetic resonance signal spectrum

In the experiment, we conducted two experiments to obtain the following image:

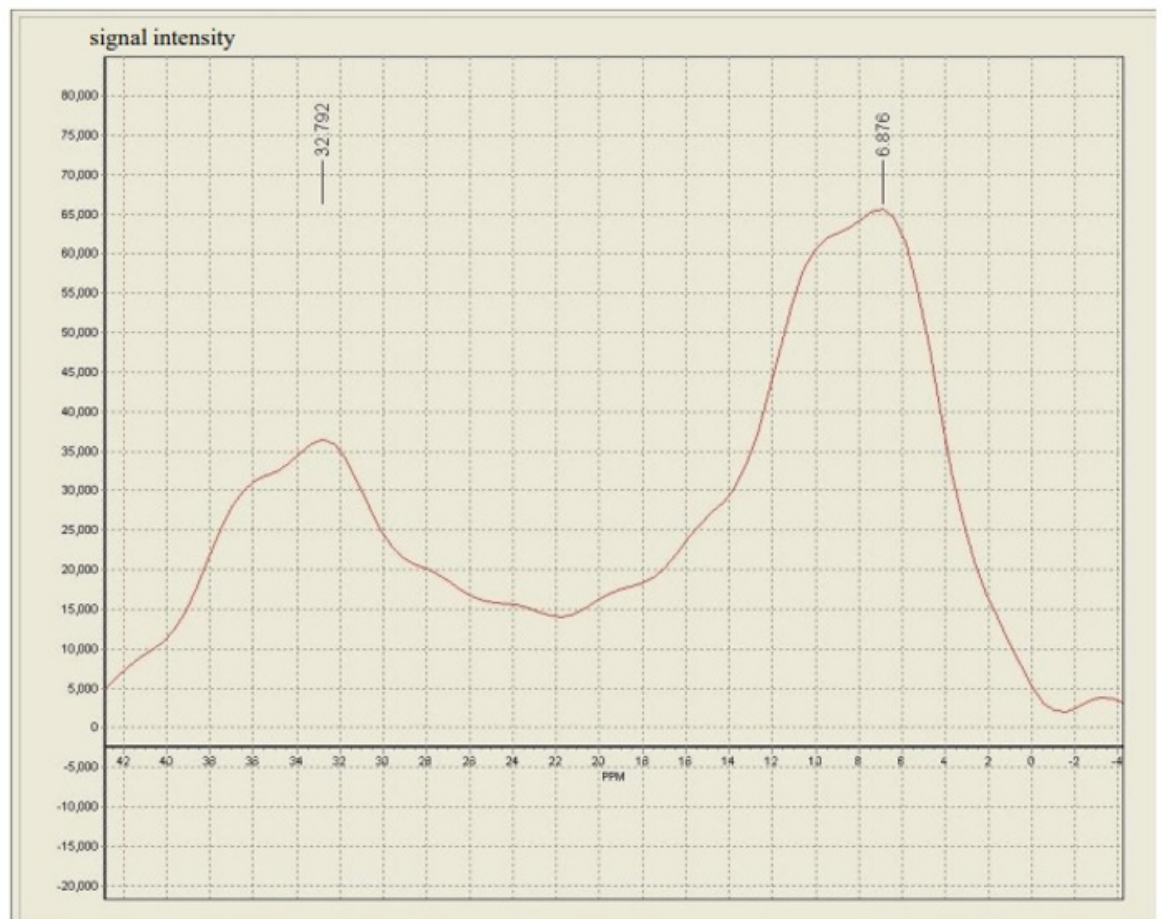

Fig.2 Measurement of chemical shift of ethanol 


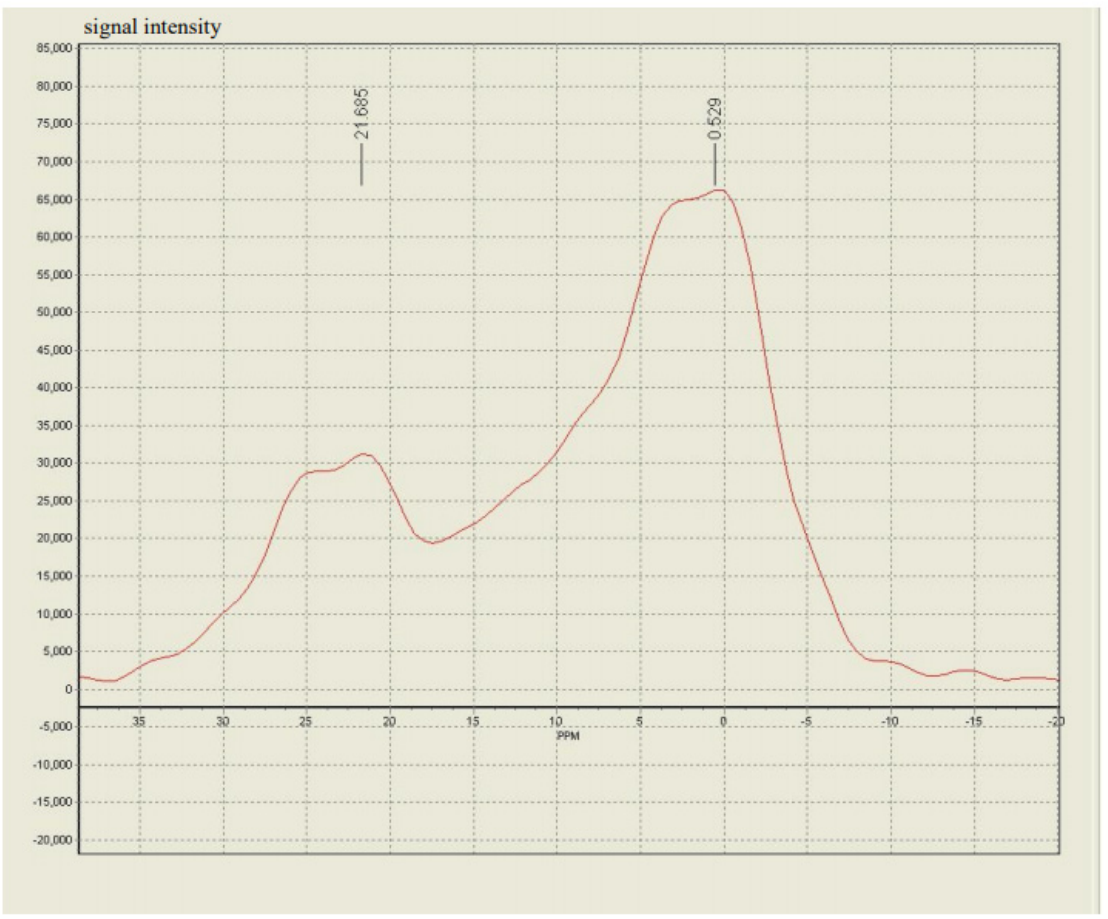

Fig .3 Measurement of chemical shift of ethanol

After many experiments, the resulting image always has only two peaks.

The reason is that the magnetic field of the experimental instrument is not uniform enough, and its resolution cannot reach to distinguish the two peaks near $32.792 \mathrm{ppm}$ in the figure.

For these two peaks, the experimental data is as follows:

Table 1. Experimental data of two peaks

\begin{tabular}{|c|c|c|c|c|c|c|}
\hline & \multicolumn{3}{|c|}{$\begin{array}{l}\text { After applying mechanical and } \\
\text { electronic shimming }\end{array}$} & \multicolumn{3}{|c|}{ After changing the electronic shim knob } \\
\hline & Peak A & Peak B & Peak C & Peak A & Peak B & Peak C \\
\hline $\begin{array}{l}\text { Frequency } \\
\text { offset }(\mathrm{KHz})\end{array}$ & -0.598 & 0 & none & -0.500 & -0.012 & none \\
\hline $\begin{array}{l}\text { Chemical } \\
\text { shift (ppm) }\end{array}$ & 32.792 & 6.876 & & 21.685 & 0.529 & \\
\hline $\begin{array}{l}\text { Peak } \\
\text { value } / 10^{3}\end{array}$ & 34.5 & 63 & & 31 & 66 & \\
\hline $\begin{array}{l}\text { Full Width } \\
\text { at Half } \\
\text { Maximum }\end{array}$ & $\begin{array}{l}0.293 \mathrm{KHz} \\
(12.17 \mathrm{ppm})\end{array}$ & $\begin{array}{l}0.232 \mathrm{KHz} \\
(9.52 \mathrm{ppm})\end{array}$ & & $\begin{array}{l}0.256 \mathrm{KHz} \\
(10.58 \mathrm{ppm})\end{array}$ & $\begin{array}{l}0.305 \mathrm{KHz} \\
(11.11 \mathrm{ppm})\end{array}$ & \\
\hline Resolution & $12.17 \mathrm{ppm}$ & $9.52 \mathrm{ppm}$ & & $10.58 \mathrm{ppm}$ & $11.11 \mathrm{ppm}$ & \\
\hline
\end{tabular}

(Note: There are two definitions of resolution in nuclear radiation detection. The first is energy resolution $\Delta \mathrm{E}=$ FWHM; the other is energy resolution $\eta=\frac{\Delta E}{E}$, Here we choose the first expression.)

Referring to the relevant literature, the typical value of the chemical shift of ethanol is about $3 \mathrm{ppm}$, but in fact, the laboratory resolution is more than $10 \mathrm{ppm}$, so it is impossible to accurately measure. For the two peaks measured, they are much greater than $3 \mathrm{ppm}$, and even reach $32 \mathrm{ppm}$, presumably due to the uniformity of the magnetic field (the uniformity is reflected in the resolution to some extent).

The structure of ethanol is shown below. 


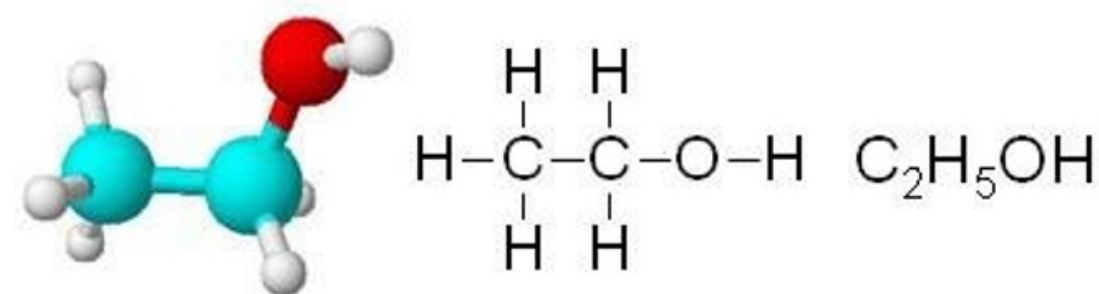

Fig .4 Structure of ethanol

Therefore, there are three kinds of hydrogen in ethanol, and the number is 3:2:1. In chemical analysis, hydrogen atoms are magnetic and electromagnetic waves are irradiated to hydrogen nuclei. It can absorb electromagnetic wave energy through resonance and undergo a transition. The relevant signals can be recorded with a nuclear magnetic resonance instrument. Hydrogen atoms in different environments absorb electromagnetic waves at different frequencies due to resonance and have different positions on the spectrum. Using information such as chemical shifts, peak area and integrated value, and coupling constants, the position of the carbon skeleton can be estimated.

Consequently, in the NMR hydrogen spectrum, the number of characteristic peaks reflects the type of chemical environment of hydrogen atoms in organic molecules; the intensity ratio of different characteristic peaks (and the height ratio of characteristic peaks) reflects the ratio of the number of hydrogen atoms in different chemical environments.

According to the data in the table, the approximate intensity ratio of peaks $\mathrm{A}$ and $\mathrm{B}$ is $1: 2$. It is speculated that peak $\mathrm{B}$ represents hydrogen in $-\mathrm{CH}_{2}$ and peak $\mathrm{A}$ is hydrogen in $-\mathrm{OH}$.

\subsection{Relationship between the concentration of ethanol solution and the lateral relaxation time $T_{2}$}

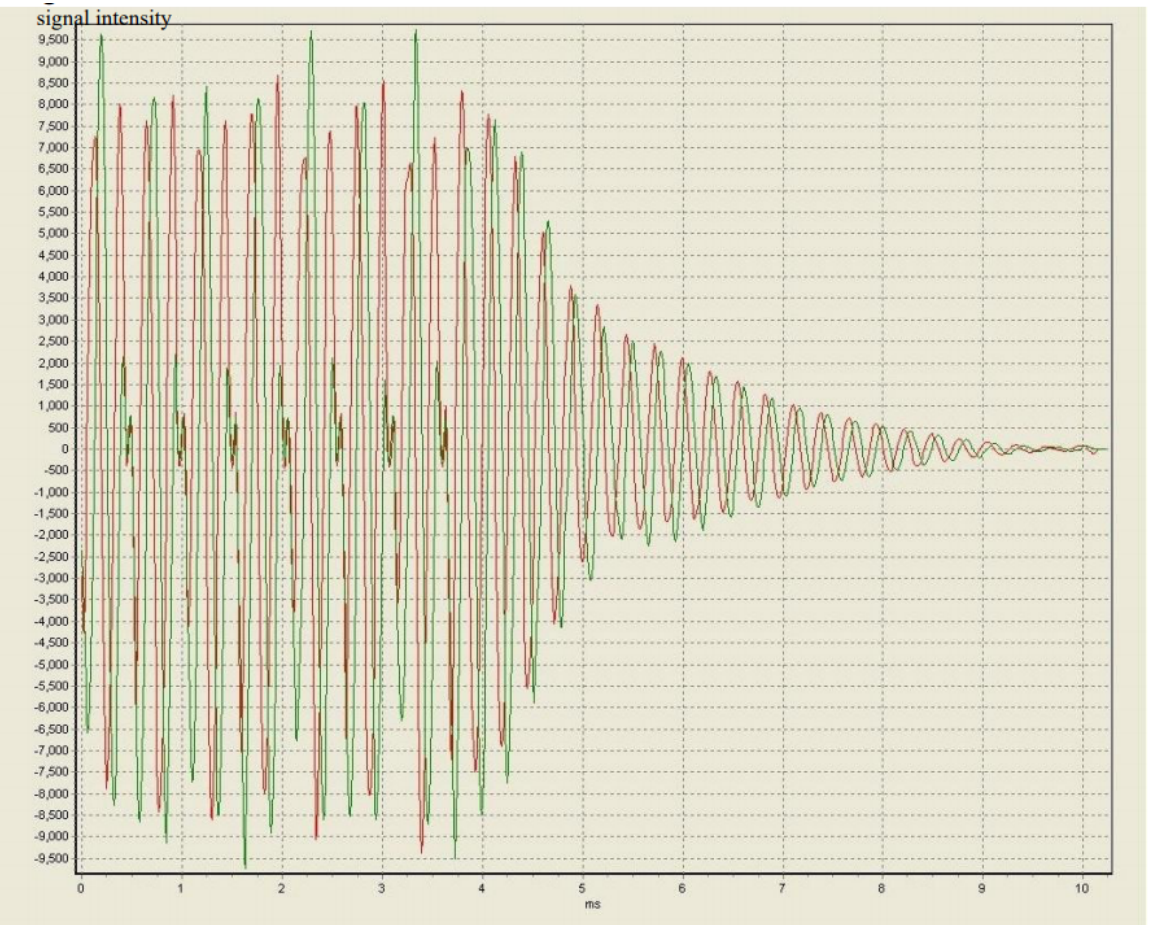

Fig. 5 Using CPMG to measure the transverse relaxation time of ethanol 
It was later known that this image appeared because the lateral relaxation time of ethanol was $1909.76 \mathrm{~ms}$, and the scan time of the aqueous ethanol solution was more than 2000. The scan time should be above 2500 . But our experimental instruments cannot bear the relaxation time of more than $1000 \mathrm{~ms}$ (there may be a crash).

Therefore, with pure ethanol solution, on the one hand, the signal intensity is low, on the one hand, the lateral relaxation time is too long, so this experimental scheme is not feasible for laboratory instruments.

By consulting the literature, we have obtained the following information- "Adding $\mathrm{CuCl}_{2} \cdot 2 \mathrm{H}_{2} \mathrm{O}$ to ethanol gasoline solution can greatly reduce the lateral relaxation time of the sample"[12], and at the same time, copper ions have the effect of amplifying the local magnetic field, Echo signals can be enhanced.

We improved the experimental scheme and configured copper sulfate ethanol aqueous solution (the copper sulfate mass fraction is constant, and the ethanol content in the ethanol aqueous solution was tested from 5\% to $40 \%$ ).

The resulting experimental table is shown below-

Table 2. Signal intensity and relaxation time of different concentrations of copper sulfate ethanol solution

\begin{tabular}{|l|l|l|}
\hline Solution concentration (\%) & Signal intensity (*1000) & $\mathrm{T}_{2}(\mathrm{~ms})$ \\
\hline 5 & 2400 & 8.42 \\
\hline 10 & 2250 & 17.02 \\
\hline 15 & 2150 & 23.75 \\
\hline 20 & 1840 & 24.26 \\
\hline 25 & 1620 & 30.07 \\
\hline 30 & 1600 & 40.35 \\
\hline 35 & 1060 & $\begin{array}{l}\text { A large amount of flocculent precipitation (copper sulfate } \\
\text { precipitation) was generated, the experiment was stopped, and the } \\
\text { concentration no longer increased. }\end{array}$ \\
\hline 40 & & \\
\hline
\end{tabular}

It can be seen that both the signal intensity (about 100 times before) and the measurement of the lateral relaxation time have been greatly improved. From the above data, an image of lateral relaxation time and ethanol solution concentration (Gaussian fitting is used) is made.

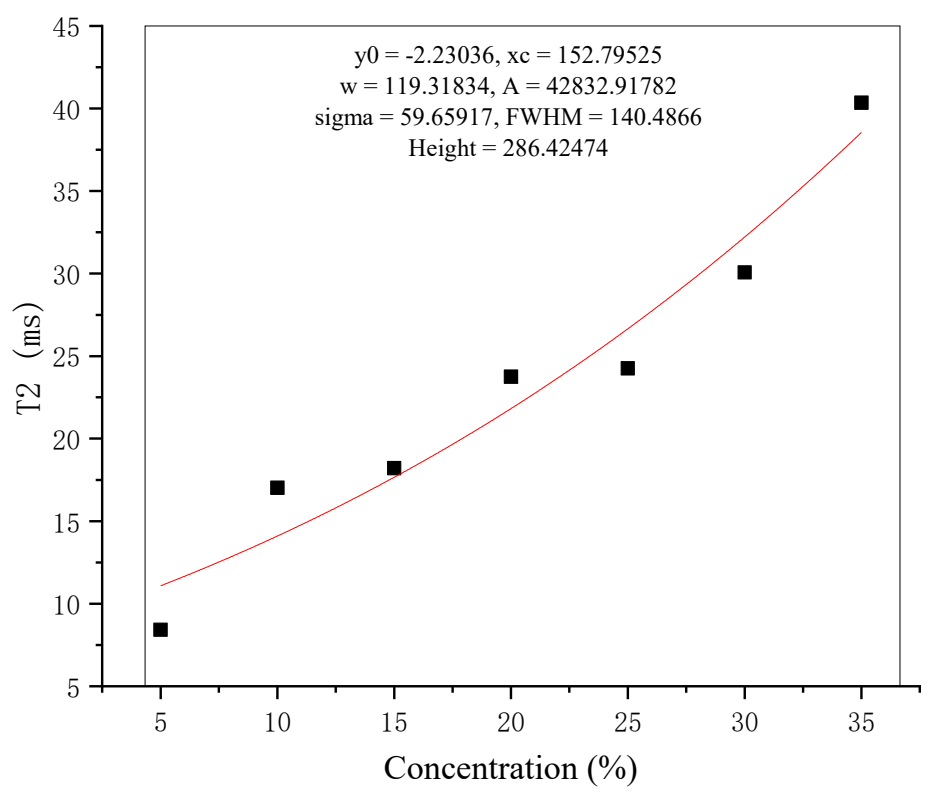

Fig. 6 Gaussian fitting of transverse relaxation time of ethanol solutions with different concentrations (addition of 5\% $\mathrm{CuSO}_{4}$ ) 
The empirical formula obtained is:

$T_{2}=-5.86+101371 * \exp \left(-0.5 *\left(\frac{x-530.23}{125.94}\right)^{\wedge} 2\right)$
Comparing the experimental results of [12], we found a big difference.

Experimental results of [12]:

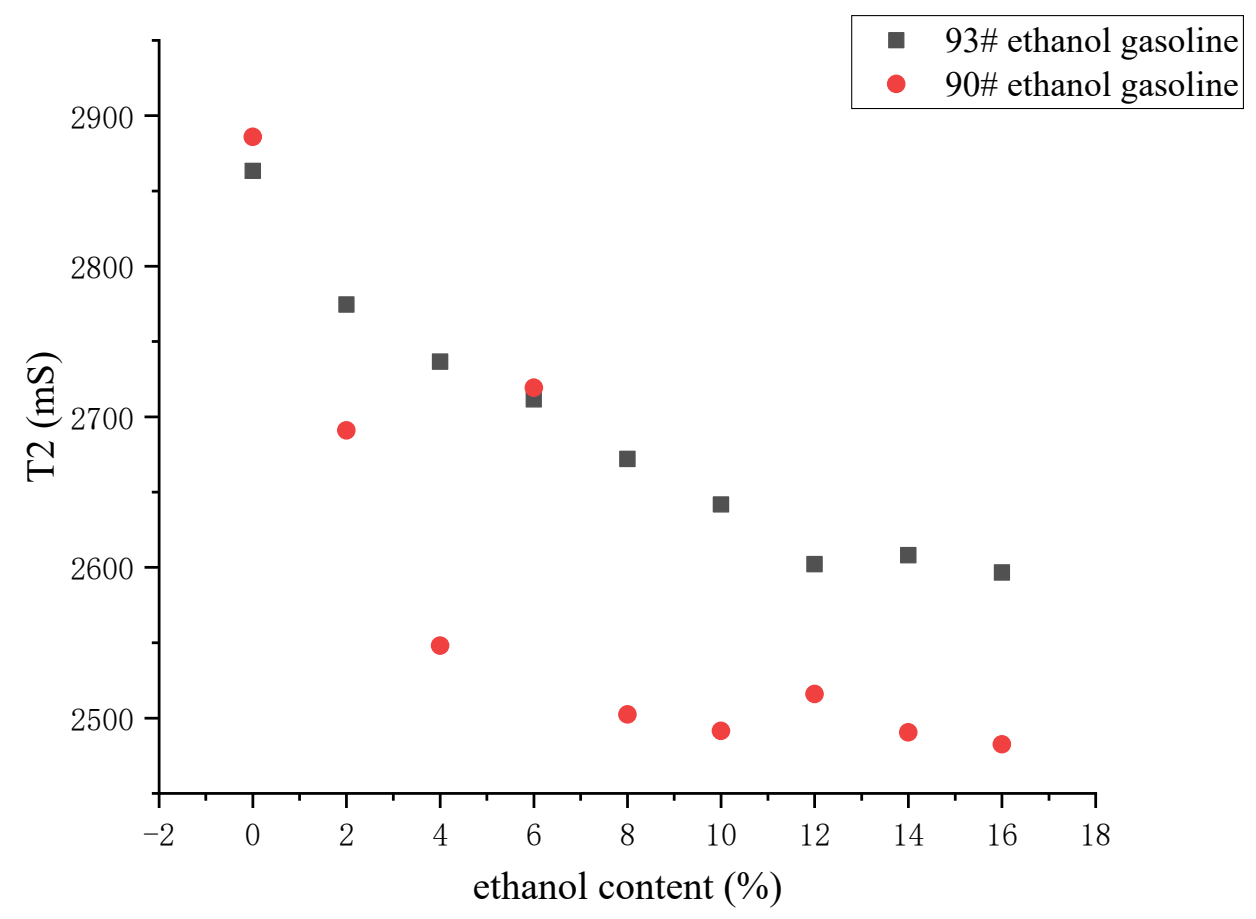

Fig. 7 Lateral relaxation time of $93 \#$ ethanol gasoline $90 \#$ ethanol gasoline after adding $\mathrm{CuCl}_{2} \cdot 2 \mathrm{H}_{2} \mathrm{O}$

It is obvious that the result obtained in [12] is that the lateral relaxation time decreases with increasing ethanol concentration. This is contrary to the results of our experiment.

There is such a big gap between the experimental results, I will analyze this as follows-

It should be noticed that the solution used in this experiment is a solution of $\mathrm{CuSO}_{4}$, and the reference [12] uses $\mathrm{CuCl}_{2}$. According to the literature, there are some differences in the chemical properties of the two- $-\mathrm{CuCl}_{2}$ is easily soluble in ethanol (solubility is $53 \mathrm{~g} / 100 \mathrm{~mL})(15$ degrees Celsius)), and $\mathrm{CuSO}_{4}$ is difficult to dissolve in ethanol (water solubility at $25^{\circ} \mathrm{C}$ is $23.05 \mathrm{~g}$, insoluble in ethanol and ether, easily soluble in water).

This is consistent with the experiment, when the ethanol concentration increased to $40 \%$, a large amount of flocculent precipitation $\left(\mathrm{CuSO}_{4}\right.$ precipitation) phenomenon is consistent. It is worth noting that, according to the literature on the effect of copper ions, the copper ions amplify the local magnetic field, that is, when the hydrogen nuclei in the material are close to each other and are tightly bound, they can only amplify the magnetic field.

In the experiment in [12], $\mathrm{CuCl}_{2}$ is used, which is easily soluble in ethanol and soluble in water, so copper ions and ethanol and water are tightly combined; but in $\mathrm{CuSO}_{4}$, copper ions in $\mathrm{CuSO}_{4}$ should not be combined with ethanol, Copper ions are more likely to combine with water and amplify the local magnetic field of the hydrogen nucleus in the water. As the ethanol concentration increases, the water content decreases, and the hydrogen nucleus used for copper ion amplification decreases. In other words, the degree of relaxation time is reduced weakening; therefore, as the concentration of the ethanol solution increases, the relaxation time increases.

At the same time, strong hydrogen bonds are easily formed between ethanol and water. The presence of ethanol will inhibit the combination of water and copper ions, and it will also weaken the decrease of relaxation time and increase the relaxation time.

\subsection{Relationship between ethanol solution concentration and nuclear magnetic resonance signal intensity}

In the ethanol molecule, the mass fraction of hydrogen is $13 \%$, and the mass fraction of hydrogen in water is $11 \%$. As the ethanol concentration increases, the hydrogen mass fraction will increase, that is, the mass of the hydrogen nucleus will increase under the same mass, and the nuclear magnetic resonance signal; theoretically, it should increase as its concentration increases.

However, with the addition of $5 \%$ copper sulfate, we got the opposite result. 
Table 3. Relationship between ethanol solution concentration and NMR signal intensity

\begin{tabular}{|l|l|l|l|l|l|l|l|l|}
\hline $\begin{array}{l}\text { Solution } \\
\text { concentration/\% }\end{array}$ & 5 & 10 & 15 & 20 & 25 & 30 & 35 & 40 \\
\hline $\begin{array}{l}\text { Signal } \\
\text { intensity/1000 }\end{array}$ & 2400 & 2250 & 2150 & 1840 & 1620 & 1600 & 1060 & $\begin{array}{l}\text { Copper } \\
\text { sulfate } \\
\text { precipitate } \\
\text { out. }\end{array}$ \\
\hline
\end{tabular}

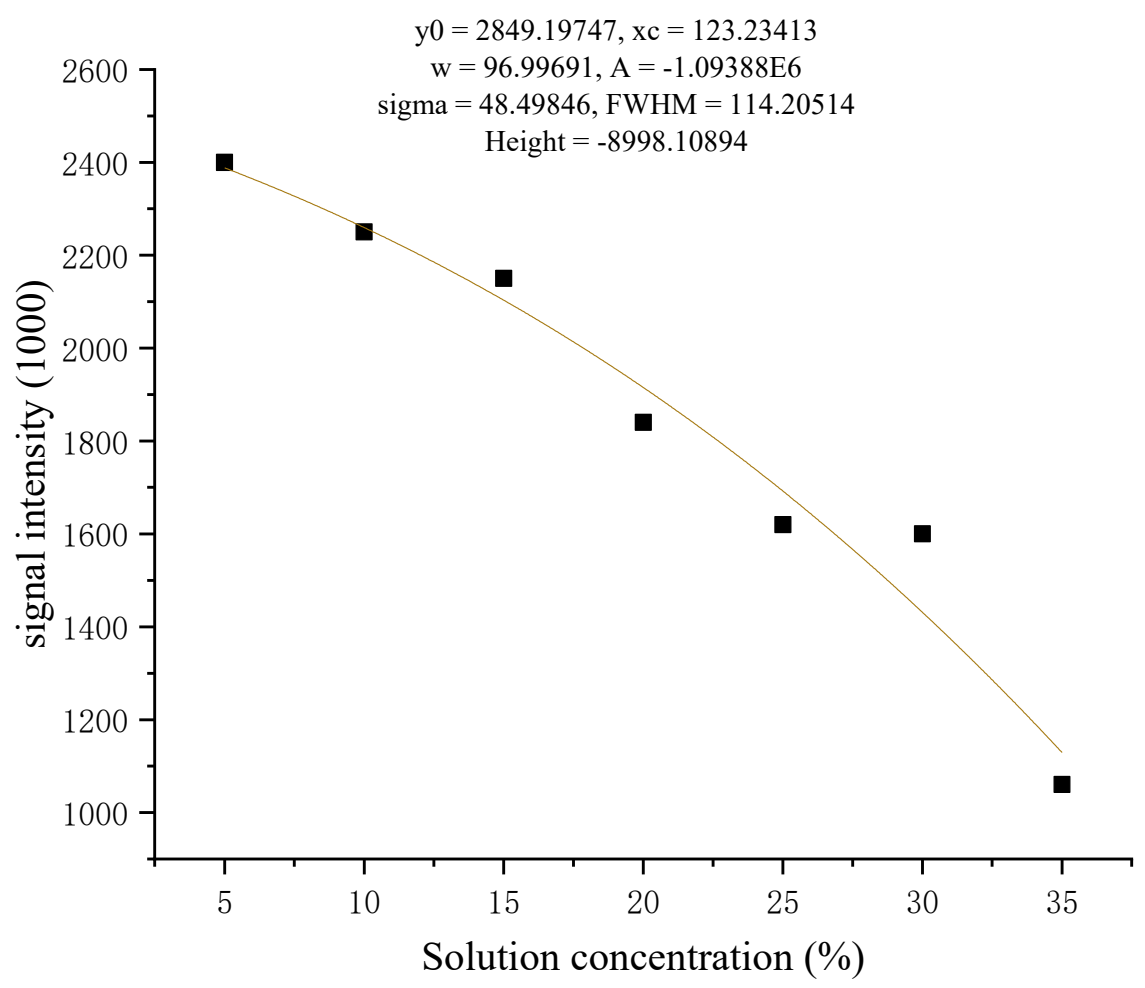

Fig. 8 Relationship between signal intensity and ethanol concentration in aqueous ethanol solution (addition of $5 \%$ copper sulfate)

Gaussian fitting it, the empirical formula of the signal intensity and ethanol concentration is

$\mathrm{I}=-29170+\left(1.2 * \frac{10^{7}}{302.5 * \operatorname{sqr}\left(\frac{P I}{2}\right)}\right) \exp \left(-2 *\left(\frac{x+10.9}{302}\right)^{\wedge} 2\right)$

The physical explanation of the phenomenon is as follows-

Due to the addition of copper ions, it has the effect of amplifying the local magnetic field; theoretically, the greater the magnetic field strength, the stronger the echo signal. The effect of copper ions can amplify the signal, which is consistent with the experimental data (compared with the addition of 5\% copper sulfate in ethanol solution and no copper sulfate in ethanol solution, the signal intensity is amplified to a hundred times).

However, as in the analysis of Experiment 11, due to the special nature of $\mathrm{CuSO}_{4}$, the increase in the ethanol content in the ethanol aqueous solution will combine with the hydrogen bond of the water, which will inhibit the amplification of the local magnetic field of the hydrogen nucleus of the copper ion by the copper ion, so the signal intensity decreases with increasing ethanol content. This factor is obviously much larger than the signal difference caused by the hydrogen nucleus mass fraction of water and ethanol hydrogen nucleus mass fraction analyzed previously, so the experimental phenomenon is contrary to the expected result.

\section{Conclusion}

In this experiment, the chemical shift of ethanol and the properties of ethanol solution were studied using the nuclear magnetic resonance instrument of Tsinghua University Modern Physics Experiment Center.

Because the resolution of the NMR instrument is greater than the chemical shift of ethanol, the expected three peaks of ethanol are not measured. However, according to the experimental results (two peaks), the 
signal intensity is proportional to the number of hydrogen nuclei, and the peak reflects Type of hydrogen nucleus-Presumably, peak B represents hydrogen in $\mathrm{CH}_{2}$, and peak A represents hydrogen in -OH.

The measurement of the transverse relaxation time $T_{2}$ of the ethanol aqueous solution cannot be completed by this nuclear magnetic resonance instrument. After changing the experimental scheme, adding copper sulfate to the ethanol aqueous solution, using copper ions to amplify the local magnetic field, the signal is strengthened (strengthened by 1 to 2 orders of magnitude), and the relaxation time decreases (decreased by about two orders of magnitude), which allows the experiment to proceed.

Finally, the empirical formula of the lateral relaxation time and ethanol concentration of the ethanol solution with a mass fraction of $5 \%$ is obtained

$$
T_{2}=-5.86+101371 * \exp \left(-0.5 *\left(\frac{x-530.23}{125.94}\right) \wedge 2\right)
$$

And the empirical formula of signal strength and ethanol concentration:

$\mathrm{I}=-29170+\left(1.2 * \frac{10^{7}}{302.5 * \operatorname{sqrt}\left(\frac{P I}{2}\right)}\right) \exp \left(-2 *\left(\frac{x+10.9}{302}\right)^{\wedge} 2\right)$

This experimental scheme uses the effect of copper ions to amplify the local magnetic field, so that in the case of low magnetic fields, it is possible to analyze the NMRrelated properties of ethanol solutions with different concentrations, which has certain practical value.

\section{References}

1. Lin Xiaoqing, Li Hong, Zhan Haolin, Du Shi jia, Huang Yu-qing, Chen Zhong. High-Resolution Pure Shift NMR Spectroscopy and Its Applications [J]. Chinese Journal of Magnetic Resonance,2019, 36(04): 425-36. (in Chinese)

2. Li Zhenwei, Hu Daoyu, Summer Liming, Study on chemical shift imaging and T2WI monitoring MR mediated absolute ethanol ablation in rabbits [J]. (in Chinese) Radiology practice, 2007, 22(04): 350-353. (in Chinese)

3. B C L. Calculating nuclear magnetic resonance chemical shifts in solvated systems [J]. Magnetic resonance in chemistry: MRC, 2020, 58(7).

4. Pennanen T, Vaara J. Nuclear Magnetic Resonance Chemical Shift in an Arbitrary Electronic Spin State [J]. Physical review letters, 2008, 100: 133002.

5. letters, 2008, 100: 133002.

6. Marell D J, Emond S J, Kulshrestha A, Hoye T R. Analysis of seven-membered lactones by computational nmr methods: Proton NMR chemical shift data are more discriminating than carbon [J]. Journal of Organic Chemistry, 2014, 79(2): 752-758.

7. Bowen S, Hilty C. Temporal chemical shift correlations in reactions studied by hyperpolarized nuclear magnetic resonance $[\mathrm{J}]$. Analytical Chemistry, 2009, 81(11): 4543-4547.

8. Sundell G N, Vogeli B, Ivarsson, Y, Chi, C N. VOGELI B, IVARSSON Y, et al. The Sign of Nuclear Magnetic Resonance Chemical Shift Difference as a Determinant of the Origin of Binding Selectivity: Elucidation of the Position Dependence of Phosphorylation in Ligands Binding to Scribble PDZ1 [J]. Biochemistry, 2018, 57(1): 66-71.

9. Lundborg M, Widmalm, G. Structural analysis of glycans by NMR chemical shift prediction [J]. Analytical Chemistry, 2011, 83(5): 1514-1517.

10. Zarzycki P, Rustad, James R. Theoretical determination of the NMR spectrum of liquid ethanol [J]. Journal of Physical Chemistry A, 2009, 113(1): 291-297.

11. Ghanghas R, Jindal A, Vasudevan S. Geometry of Hydrogen Bonds in Liquid Ethanol Probed by Proton NMR Experiments [J]. Journal of Physical Chemistry B, 2020, 124(4): 662-667.

12. Bai Huaiyong, Zhou Ge, Wang Diansheng. Experimental study on NMR of different concentrations of ethanol-water solution [J]. Experimental Technology and Management, 2013, 30(08): 39-42. (in Chinese)

13. Zhou Ge, Bai Huaiyong, Wang Diansheng. Experimental Investigation on NMR Detection of Ethanol Content in Ethanol Gasoline [J]. (in Chinese) Laboratory Research and Explore. (in Chinese) 